\title{
ON THE SENSITIVITY ANALYSIS OF ENERGY QUANTO OPTIONS.
}

\author{
RODWELL KUFAKUNESU AND FARAI JULIUS MHLANGA
}

\begin{abstract}
In recent years there has been an advent of quanto options in energy markets. The structure of the payoff is rather a different type from other markets since it is written as a product of an underlying energy index and a measure of temperature. In the HJM framework, by adopting the futures energy dynamics, we use the Malliavin calculus to derive the delta and the cross-gamma expectation formulas. This work can be viewed as an extension of the work done, for example by Benth et al. [1.
\end{abstract}

\section{INTRODUCTION}

The paper investigates hedging of the energy quanto options using the Malliavin Calculus approach by Nualart [2]. This method has shown that it outperforms the finite difference approach when it comes to discontinuous payoffs, see Benth et al. 33. Quanto options in the equity market differ from those designed for energy markets by the structure of their payoffs. The energy quanto option has a product payoff which is structured in such a way that it takes advantage of the high correlation between energy consumption and certain weather conditions thereby enabling price and weather risk to be controlled simultaneously, refer Caporin et al. 44. On the other hand, the equity quanto has a normal structure. Ho et al. [5] noted that quanto options, in general, are better hedgers than a simple combination of plain vanilla options. In energy markets, they give exposure to the volumetric risk input of weather conditions on energy prices, see Zhang [6].

Heath et al. [7] introduced the so-called Hearth-Jarrow-Merton (HJM) approach in fixed income markets where the dynamics of the forward rates are directly specified, see Benth et al. 88. The fact that most contracts in energy markets are settled in futures and forward, the framework was later on in 2000 adopted in this market by Clewlow and Strickland [9]. There have been few papers in literature analysing the hedging of this quanto option product. Benth et al. 1] recently studied the pricing and hedging of quanto energy options in this framework basing on both the spot and the futures products as the underlying processes. The authors derived analytic expressions for the energy delta, the temperature delta, and the cross-gamma hedging. If the payoff functions are discontinuous then their hedging method fails. In this paper, we derive the so-called derivative free hedging formulae using a much more powerful tool: the Malliavin calculus. The Malliavin calculus technique has been used by many authors in hedging equity derivative products, see for example, (Benth et al. 8, Benth et al. [10, Di Nunno et al. [11, Fournié et al. [12, 13], Karatzas et al. [14], Mhlanga [15]). In all these references the methods were not

Date: October 16, 2018.

Key words and phrases. energy option, futures, Malliavin derivatives, HJB framework . 
applied in a product payoff structure such as ours and with an interval delivering period. Our results can be viewed as an extension of Benth et al. [1].

We only focus on deriving the delta and the cross-gamma hedging expectation formulae. The paper is organised as follows. Section 2, reviews the structure of the quanto option as in Benth et al. [1]. We present the futures asset dynamics general diffusion models under the Heath-Jarrow-Morton (HJM) framework. In Section 3, we review the necessary tools from Malliavin calculus to be applied in our proofs. In Section 4, the 'Greeks' that is, the delta and the cross-gamma are derived in the independent case, when the correlation value is zero and in Section 5 the correlation case, the Greek formulae are obtained. The residual risk is also discussed. Some examples are provided in Section 6. Finally, in Section 7, we conclude.

\section{The Contract Structure and Pricing of Quanto Options}

In this section, we review the commodity quanto pricing, see, for example, Benth et al. [1] and in particular, we follow their notations therein. The quanto has a payoff function $S$ given by:

$$
S=\left(T_{v a r}-T_{f i x}\right) \times\left(E_{v a r}-E_{f i x}\right),
$$

where $T_{\text {var }}$ represents some variable temperature measure, $T_{\text {fix }}$ represents some fixed temperature measure, and $E_{v a r}, E_{f i x}$ are the variable and fixed energy price, respectively. To avoid the downside risk on this quanto contract it has been reported in Benth et al. [1] that for hedging purposes, it is reasonable to buy a contract with optionality. In the temperature market of Chicago Mercantile Exchange (CME), for contracts are written on the aggregated amount of heating-degree days (HDD) and cooling-degree days (CDD). The temperature index is used as the underlying. The HDD (similarly the CDD) over a measurement period $\left[\tau_{1}, \tau_{2}\right]$ is defined by:

$$
I_{\left[\tau_{1}, \tau_{2}\right]}:=H D D(t)=\max (c-T(t), 0),
$$

where $T(t)$ is the mean temperature on day $t$, and $c$ is the pre-specified temperature threshold (eg., $65^{0} \mathrm{~F}$ or $18^{\circ} \mathrm{C}$ ). If the contract is specified as the accumulated HDD over $\left[\tau_{1}, \tau_{2}\right]$ we have :

$$
I_{\left[\tau_{1}, \tau_{2}\right]}:=\sum_{t=\tau_{1}}^{\tau_{2}} H D D(t)=\sum_{t=\tau_{1}}^{\tau_{2}} \max (c-T(t), 0),
$$

analogously for CDD.

We note that quanto options have a payoff function that is a function of two underlying assets, temperature and price. We focus on quanto options with payoff function $f(E, I)$ where $E$ is an index of the energy price and $I$ is an index of temperature. The energy index $E$ over a period $\left[\tau_{1}, \tau_{2}\right]$ is given as an average spot price by:

$$
E=\frac{1}{\tau_{2}-\tau_{1}} \sum_{u=\tau_{1}}^{\tau_{2}} S_{u}
$$


where $S_{u}$ is the energy spot price. In addition, we assume that the temperature index is defined by

$$
I=\sum_{u=\tau_{1}}^{\tau_{2}} g\left(T_{u}\right)
$$

where $T_{u}$ denotes the temperature at time $u$ and $g$ some function. For example, for a quanto option involving the HDD index, we choose $g(x)=\max (x-18,0)$. To price the quanto option exercised at the time $\tau_{2}$, its arbitrage-free price at time $t \leq \tau_{2}$ becomes

$$
C_{t}=e^{-r\left(\tau_{2}-t\right)} \mathbb{E}_{t}^{\mathbb{Q}}\left[f\left(\frac{1}{\tau_{2}-\tau_{1}} \sum_{u=\tau_{1}}^{\tau_{2}} S_{u}, \sum_{u=\tau_{1}}^{\tau_{2}} g\left(T_{u}\right)\right)\right],
$$

where $r>0$ represents a constant risk-free interest rate and $\mathbb{E}_{t}^{\mathbb{Q}}$ is the expectation operator with respect to $\mathbb{Q}$, conditioned on the market information at time $t$ given by the filtration $\mathcal{F}_{t}$. Following [1]'s argument on the relationship between the quanto option and the futures contract on the energy ad temperature indexes $E$ and $I$, we note that the price at time $t \leq \tau_{2}$ of a futures contract written on some energy price with delivery period $\left[\tau_{1}, \tau_{2}\right]$ is given by

$$
F^{E}\left(t ; \tau_{1}, \tau_{2}\right)=\mathbb{E}_{t}^{\mathbb{Q}}\left[\frac{1}{\tau_{2}-\tau_{1}} \sum_{u=\tau_{1}}^{\tau_{2}} S_{u}\right]
$$

At $t=\tau_{2}$ we have:

$$
F^{E}\left(\tau_{2} ; \tau_{1}, \tau_{2}\right)=\frac{1}{\tau_{2}-\tau_{1}} \sum_{u=\tau_{1}}^{\tau_{2}} S_{u}
$$

This means that the future prices is exactly equal to what is being delivered. Applying the same argument to the temperature index, with price dynamics denoted by $F^{I}\left(t, \tau_{1}, \tau_{2}\right)$, the quanto option price $C_{t}$ can be written as:

$$
\begin{aligned}
C_{t} & =e^{-r\left(\tau_{2}-t\right)} \mathbb{E}_{t}^{\mathbb{Q}}\left[f\left(\frac{1}{\tau_{2}-\tau_{1}} \sum_{u=\tau_{1}}^{\tau_{2}} S_{u}, \sum_{u=\tau_{1}}^{\tau_{2}} g\left(T_{u}\right)\right)\right] \\
& =e^{-r\left(\tau_{2}-t\right)} \mathbb{E}_{t}^{\mathbb{Q}}\left[f\left(F^{E}\left(\tau_{2} ; \tau_{1}, \tau_{2}\right), F^{I}\left(\tau_{2} ; \tau_{1}, \tau_{2}\right)\right)\right] .
\end{aligned}
$$

The advantage of writing the quanto option price as in Eq.(2.8) is that futures are traded financial assets. Let $\bar{K}_{E}, \bar{K}_{I}$ denote the high strikes for the energy and temperature indexes, respectively and $\underline{K}_{E}, \underline{K}_{I}$ denote the low strikes for the energy and temperature indexes, respectively. Now we can define the payoff function

$$
p\left(F^{E}\left(\tau_{2} ; \tau_{1}, \tau_{2}\right), F^{I}\left(\tau_{2} ; \tau_{1}, \tau_{2}\right), \bar{K}_{E}, \bar{K}_{I}, \underline{K}_{E}, \underline{K}_{I}\right):=p
$$

so that

$$
\begin{aligned}
p & =\alpha \times\left[\max \left(F^{E}\left(\tau_{2} ; \tau_{1}, \tau_{2}\right)-\bar{K}_{E}, 0\right) \times \max \left(F^{I}\left(\tau_{2} ; \tau_{1}, \tau_{2}\right)-\bar{K}_{I}, 0\right)\right. \\
& \left.+\max \left(\underline{K}_{E}-F^{E}\left(\tau_{2} ; \tau_{1}, \tau_{2}\right), 0\right) \times \max \left(\underline{K}_{I}-F^{I}\left(\tau_{2} ; \tau_{1}, \tau_{2}\right), 0\right)\right]
\end{aligned}
$$


where $\alpha$ is the contractual volume adjustment factor. As in Benth et al. [1, for illustration purposes, we consider the product call structure with the volume adjuster $\alpha$ normalized to 1, that is, we consider the price of an option with the following payoff function:

$$
\hat{p}=\max \left(F^{E}\left(\tau_{2} ; \tau_{1}, \tau_{2}\right)-\bar{K}_{E}, 0\right) \times \max \left(F^{I}\left(\tau_{2} ; \tau_{1}, \tau_{2}\right)-\bar{K}_{I}, 0\right),
$$

and the quanto option at time $t$ is given by:

$$
C_{t}=e^{-r\left(\tau_{2}-t\right)} \mathbb{E}_{t}^{\mathbb{Q}}\left[\hat{p}\left(F^{E}\left(\tau_{2} ; \tau_{1}, \tau_{2}\right), F^{I}\left(\tau_{2} ; \tau_{1}, \tau_{2}\right), \bar{K}_{E}, \bar{K}_{I}\right)\right] .
$$

2.1. The Asset Dynamics. We use the HJM risk-neutral dynamics of the forward contract at time $t$. Consider the general diffusion futures model under the risk-neutral measure $\mathbb{Q}$ be given as :

$$
\begin{aligned}
d F^{E}\left(t ; \tau_{1}, \tau_{2}\right) & =\sigma_{E}\left(t, F^{E}\left(t ; \tau_{1}, \tau_{2}\right)\right) d W^{E}(t), \\
d F^{I}\left(t ; \tau_{1}, \tau_{2}\right) & =\sigma_{I}\left(t, F^{I}\left(\tau ; \tau_{1}, \tau_{2}\right)\right) d W^{I}(t) .
\end{aligned}
$$

with $F^{E}\left(0 ; \tau_{1}, \tau_{2}\right)>0$ and $F^{I}\left(0 ; \tau_{1}, \tau_{2}\right)>0$ where $\sigma_{E}, \sigma_{I}$ are deterministic volatilities and $W^{E}, W^{I}$ are correlated Brownian motions with a correlation parameter $\rho \in(-1,1)$. The process $F^{E}$ is the option price of a future contact written on some energy price and $F^{I}$ is the option price of a future contact written on some temperature price.

Given an arbitrary $W^{E}$, there exists $\widetilde{W}^{I}$ which is independent of $W^{E}$ and $W^{I}$. Then, we can express $W^{I}$ as follows

$$
W^{I}=\rho W^{E}+\sqrt{1-\rho^{2} W^{I}} .
$$

Thus we have

$$
\begin{aligned}
& d F^{E}\left(t ; \tau_{1}, \tau_{2}\right)=\sigma_{E}\left(t, F^{E}\left(t ; \tau_{1}, \tau_{2}\right)\right) d W^{E}(t) \\
& d F^{I}\left(t ; \tau_{1}, \tau_{2}\right)=\rho \sigma_{I}\left(t, F^{I}\left(t ; \tau_{1}, \tau_{2}\right)\right) d W^{E}(t)+\sigma_{I}\left(t, F^{I}\left(t ; \tau_{1}, \tau_{2}\right)\right) \sqrt{1-\rho^{2}} d \widetilde{W}^{I}(t)
\end{aligned}
$$

The above equations can be written in matrix form as follows:

$$
\left(\begin{array}{c}
d F^{E} \\
d F^{I}
\end{array}\right)=\left(\begin{array}{cc}
\sigma_{E}\left(t, F^{E}\right) & 0 \\
\rho \sigma_{I}\left(t, F^{I}\right) & \sigma_{I}\left(t, F^{I}\right) \sqrt{1-\rho^{2}}
\end{array}\right)\left(\begin{array}{c}
d W^{E} \\
d \widetilde{W}^{I}
\end{array}\right) .
$$

We can write this as:

$$
d \bar{F}=a\left(t, F^{I}, F^{E}\right) d \bar{W},
$$

where the matrix $a:\left(\left[0, \tau_{2}\right] \times \mathbb{R}^{2}\right) \rightarrow \mathcal{M}_{2}$, satisfies the growth and Lipschitz conditions. We can write (2.15) as:

$$
\bar{F}=\bar{F}_{0}+\int_{0}^{t} a\left(t, F^{I}, F^{E}\right) d \bar{W}, \bar{F}_{0}>\mathbf{0} .
$$

Given this dynamics the quanto option becomes :

$$
C_{t}=\mathbb{E}^{\mathbb{Q}}\left[\tilde{g}\left(\int_{0}^{\tau_{2}} \sigma_{E}\left(t, F^{E}\right) d W^{E}\right) \tilde{h}\left(\int_{0}^{\tau_{2}} \sigma_{I}\left(t, F^{I}\right) d W^{E}, \int_{0}^{\tau_{2}} \sigma_{I}\left(t, F^{I}\right) d \widetilde{W}^{I}\right)\right],
$$

where $\tilde{g}(x)=\left(x-K^{E}\right)^{+}$and $\tilde{h}(x, y)=\left(\rho x+\sqrt{1-\rho^{2}} y-K^{I}\right)^{+}$are measurable functions. 


\section{A Primer on the Malliavin Derivative Properties}

In this section, we review the necessary Malliavin derivative properties. These properties were also highlighted in Fournié et al. [12] and Mhlanga [15] and the proofs can be found in Nualart [2]. Let $\left\{W(t), 0 \leq t \leq \tau_{2}\right\}$ be an $n$-dimensional Brownian motion defined on a complete probability space $(\Omega, \mathcal{F}, \mathbb{F}, \mathbb{Q})$. Let $S$ denote the class of random variables of the form

$$
F=f\left(\int_{0}^{\tau_{2}} h_{1}(t) d W(t), \cdots, \int_{0}^{\tau_{2}} h_{n}(t) d W(t)\right), \quad f \in C^{\infty}\left(\mathbb{R}^{n}\right),
$$

where $h_{1}, \cdots, h_{n} \in L^{2}\left(\left[0, \tau_{2}\right]\right)$.

For $F \in S$, the Malliavin derivative $D F$ of $F$ is defined as the process $\left\{D_{t} F, t \in\left[0, \tau_{2}\right]\right\}$ in $L^{2}\left(\left[0, \tau_{2}\right]\right)$ is defined by :

$$
D_{t} F=\sum_{i=1}^{n} \frac{\partial f}{\partial x_{i}}\left(\int_{0}^{\tau_{2}} h_{1}(t) d W(t), \cdots, \int_{0}^{\tau_{2}} h_{n}(t) d W(t)\right) h_{i}(t), \quad t \geq 0 \text { a.s. }
$$

On $L^{2}\left(\left[0, \tau_{2}\right]\right)$ define the norm as :

$$
\|F\|_{1,2}:=\left(\mathbb{E}^{\mathbb{Q}}|F|^{2}+\mathbb{E}^{\mathbb{Q}}\left[\int_{0}^{\tau_{2}}\left|D_{t} F\right|^{2} d t\right]\right)^{\frac{1}{2}} .
$$

The chain rule holds for the Malliavin derivative in the following form.

Property P1. Let $F=\left(F_{1}, \ldots, F_{n}\right) \in \mathbb{D}^{1,2}$ and let $\varphi: \mathbb{R}^{n} \rightarrow \mathbb{R}$ be a continuously differentiable function with bounded partial derivatives. Then $\varphi(F) \in \mathbb{D}^{1,2}$ and

$$
D_{t} \varphi(F)=\sum_{i=1}^{n} \frac{\partial \varphi}{\partial x_{i}}(F) D_{t} F_{i}, t \geq 0 \text { a.s. }
$$

Property P2. Let $\left\{X_{t}, t \geq 0\right\}$ be an $\mathbb{R}^{n}$ valued Itô process whose dynamics are governed by the stochastic differential equation

$$
d X_{t}=b\left(X_{t}\right) d t+\sigma\left(X_{t}\right) d W_{t}
$$

where $b$ and $\sigma$ are supposed to be continuously differentiable functionals with bounded derivatives and $\sigma(x) \neq 0$ for all $x \in \mathbb{R}^{n}$. Let $\left\{Y_{t}, t \geq 0\right\}$ be the associated first variation process given by the stochastic differential equation

$$
d Y_{t}=b^{\prime}\left(X_{t}\right) Y_{t} d t+\sum_{i=1}^{n} \sigma_{i}^{\prime}\left(X_{t}\right) Y_{t} d W_{t}^{i}, Y_{0}=I_{n}
$$

where $I_{n}$ is the identity matrix of $\mathbb{R}^{n}$, primes denote derivatives and $\sigma_{i}$ is the $i$-th column vector of $\sigma$. The the process $\left\{X_{t}, t \geq 0\right\}$ belongs to $\mathbb{D}^{1,2}$ and its Malliavin derivative is given by

$$
D_{r} X_{t}=Y_{t} Y_{r}^{-1} \sigma\left(X_{r}\right) 1_{\{r \leq t\}}, r \geq 0 \text { a.s. }
$$

which is equivalent to

$$
Y_{t}=D_{r} X_{t} \sigma^{-1}\left(X_{r}\right) Y_{r} 1_{\{r \leq t\}} \text { a.s. }
$$


The Malliavin derivative has an adjoint operator called Skorohod integral (also known as the divergence operator $\delta$ ). We shall denote the domain of the adjoint operator $\delta$ by $\operatorname{Dom}(\delta)$.

Property P3. Let $u \in L^{2}\left(\Omega \times\left[0, \tau_{2}\right]\right)$. Then $u$ belongs to the domain $\operatorname{Dom}(\delta)$ of $\delta$ if for all $F \in \mathbb{D}^{1,2}$ we have

$$
\left|\mathbb{E}\left[\langle D F, u\rangle_{L^{2}(\Omega)}\right]\right|=\left|\mathbb{E}\left[\int_{0}^{\tau_{2}} D_{t} F u(t) d t\right]\right| \leq c\|F\|_{L^{2}(\Omega)}
$$

where $c$ is some constant depending on $u$. If $u$ belongs to $\operatorname{Dom}(\delta)$, then

$$
\delta(u)=\int_{0}^{\tau_{2}} u_{t} \delta W_{t}
$$

is the element of $L^{2}(\Omega)$ such that the integration by parts formula holds:

$$
\mathbb{E}\left[\left(\int_{0}^{\tau_{2}} D_{t} F u_{t} d t\right)\right]=\mathbb{E}[F \delta(u)] \text { for all } F \in \mathbb{D}^{1,2} .
$$

An important property of the Skorohod integral $\delta$ is that its domain $\operatorname{Dom}(\delta)$ contains all adapted stochastic processes which belong to $L^{2}\left(\Omega \times\left[0, \tau_{2}\right]\right)$. For such processes the Skorohod integral $\delta$ coincides with the Itô stochastic integral.

Property $P 4$. If $u$ is an adapted process belonging to $L^{2}\left(\Omega \times\left[0, \tau_{2}\right]\right)$, then

$$
\delta(u)=\int_{0}^{\tau_{2}} u(t) d W_{t}
$$

Further, if the random variable $F$ is $\mathcal{F}_{\tau_{2}}$-adapted and belongs to $\mathbb{D}^{1,2}$ then, for any $u$ in $\operatorname{Dom}(\delta)$, the random variable $F u$ will be Skorohod integrable.

Property P5. Let $F$ belongs to $\mathbb{D}^{1,2}$ and $u \in \operatorname{Dom}(\delta)$ such that $\mathbb{E}\left[\int_{0}^{\tau_{2}} F^{2} u_{t}^{2} d t\right]<\infty$. Then $F u \in \operatorname{Dom}(\delta)$ and

$$
\delta(F u)=F \delta(u)-\int_{0}^{\tau_{2}} D_{t} F u_{t} d t
$$

whenever the right hand side belongs to $L^{2}(\Omega)$. In particular, if $u$ is moreover adapted, we have

$$
\delta(F u)=F \int_{0}^{\tau_{2}} u_{t} d W_{t}-\int_{0}^{\tau_{2}} D_{t} F u_{t} d t
$$

\section{The Independent CASE}

From the diffusion stochastic differential equation (2.13) with $\rho=0$, consider the following HJM risk-neutral dynamics of the forward contract at time $t$. We call this the 'independent case'. Let the future price processes under the risk-neutral measure $\mathbb{Q}$ be given as

$$
d F^{i}\left(t ; \tau_{1}, \tau_{2}\right)=\sigma_{i}\left(t ; \tau_{1}, \tau_{2}\right) F^{i}\left(t ; \tau_{1}, \tau_{2}\right) d W^{i}(\tau), F^{i}\left(0 ; \tau_{1}, \tau_{2}\right)>0
$$


for $E, I=i$. The function $F^{i}\left(0 ; \tau_{1}, \tau_{2}\right)$ represents today's forward price. We call this, the independent case since $\rho=0$. Explicitly this can be written as:

$$
\begin{aligned}
& F^{E}\left(\tau_{2} ; \tau_{1}, \tau_{2}\right)=F^{E}\left(0 ; \tau_{1}, \tau_{2}\right) \exp \left(-\frac{1}{2} \int_{0}^{\tau_{2}} \sigma_{E}^{2}\left(u ; \tau_{1}, \tau_{2}\right) d u+\int_{0}^{\tau_{2}} \sigma_{E}\left(u ; \tau_{1}, \tau_{2}\right) d W^{E}(u)\right) \\
& F^{I}\left(\tau_{2} ; \tau_{1}, \tau_{2}\right)=F^{I}\left(0 ; \tau_{1}, \tau_{2}\right) \exp \left(-\frac{1}{2} \int_{0}^{\tau_{2}} \sigma_{I}^{2}\left(u ; \tau_{1}, \tau_{2}\right) d u+\int_{0}^{\tau_{2}} \sigma_{I}\left(u ; \tau_{1}, \tau_{2}\right) d W^{I}(u)\right)
\end{aligned}
$$

where $W^{E}$ and $W^{I}$ are Brownian motions. Here, $\int_{0}^{\tau_{2}} \sigma_{i}^{2}\left(\tau ; \tau_{1}, \tau_{2}\right) d \tau<\infty$ meaning $\tau \mapsto$ $F^{i}\left(\tau ; \tau_{1}, \tau_{2}\right)$ is a martingale. Introduce $g: \mathbb{R} \mapsto \mathbb{R}$ and $h: \mathbb{R} \mapsto \mathbb{R}$ measurable functions. The payoff structure of a quanto option on the forwards with maturity at time $\tau_{2}$ given by

$$
C=\mathbb{E}^{\mathbb{Q}}\left[g\left(F^{E}\left(\tau_{2} ; \tau_{1}, \tau_{2}\right)\right) h\left(F^{I}\left(\tau_{2} ; \tau_{1}, \tau_{2}\right)\right)\right],
$$

where $g(x)=\left(x-K^{E}\right)^{+}$and $h(x)=\left(x-K^{I}\right)^{+}$and the risk-free interest rate $r=0$. We assume the following integrability conditions:

$$
\mathbb{E}\left[g^{2}\left(F^{E}\left(\tau_{2} ; \tau_{1}, \tau_{2}\right)\right)\right]<\infty, \quad \mathbb{E}\left[h^{2}\left(F^{I}\left(\tau_{2} ; \tau_{1}, \tau_{2}\right)\right)\right]<\infty .
$$

At several places, we will require the diffusion matrix $\sigma_{i}, i=E, I$ to satisfy the following condition:

$$
\exists \eta>0 \xi^{*} \sigma^{*}\left(t ; \tau_{1}, \tau_{2}\right) \sigma\left(t ; \tau_{1}, \tau_{2}\right) \xi>\eta|\xi|^{2} \text { for all } \xi \in \mathbb{R}^{n}, t \in\left[\tau_{1}, \tau_{2}\right] \text { with } \xi \neq 0 .
$$

where $\xi^{*}$ denotes the transpose of $\xi$. This is called the uniform ellipticity condition.

The weight function obtained when computing Greeks using the integration by parts formula should not degenerate with probability one, otherwise the computation will not be valid. To avoid this degeneracy we introduce the set $\Upsilon_{n}$ (see [12]) defined by

$$
\Upsilon_{n}=\left\{a \in L^{2}\left(\left[0, \tau_{2}\right]\right) \mid \int_{0}^{t_{i}} a(t) d t=1 \text { for all } i=1, \ldots, n\right\} .
$$

Proposition 4.1. Assume that the diffusion matrix $\sigma_{E}$ is uniformly elliptic. Then for all $a \in \Upsilon_{n}$, the delta of the energy option is given by :

$$
\Delta_{E}=\mathbb{E}^{\mathbb{Q}}\left[g\left(F^{E}\left(\tau_{2} ; \tau_{1}, \tau_{2}\right)\right) h\left(F^{I}\left(\tau_{2} ; \tau, \tau_{2}\right)\right) \pi^{\Delta_{E}}\right],
$$

where the Malliavin weight $\pi^{\Delta_{E}}$ is

$$
\pi^{\Delta_{E}}=\int_{0}^{\tau_{2}} a(t)\left(\sigma_{E}^{-1}\left(t ; \tau_{1}, \tau_{2}\right) Y_{E}\left(t ; \tau_{1}, \tau_{2}\right)\right)^{*} d W^{E}(t)
$$

Proof. Let $g$ be a continuously differentiable function with bounded derivatives. Introduce

$$
Y_{E}\left(t ; \tau_{1}, \tau_{2}\right)=\exp \left(-\frac{1}{2} \int_{0}^{t} \sigma_{E}^{2}\left(u ; \tau_{1}, \tau_{2}\right) d u+\int_{0}^{t} \sigma_{E}\left(u ; \tau_{1}, \tau_{2}\right) d W^{E}(u)\right) .
$$

This implies that

$$
F^{E}\left(\tau ; \tau_{1}, \tau_{2}\right)=F^{E}\left(0 ; \tau_{1}, \tau_{2}\right) Y_{E}\left(t ; \tau_{1}, \tau_{2}\right)
$$

An application of Property $P 2$ shows that $F^{E}\left(\tau_{2} ; \tau_{1}, \tau_{2}\right)$ belongs to $\mathbb{D}^{1,2}$ and we have:

$$
D_{t} F^{E}\left(\tau_{2} ; \tau_{1}, \tau_{2}\right)=Y_{E}\left(\tau_{2} ; \tau_{1}, \tau_{2}\right) Y_{E}^{-1}\left(t ; \tau_{1}, \tau_{2}\right) \sigma_{E}\left(t ; \tau_{1}, \tau_{2}\right) 1_{t<\tau_{2}} .
$$


This is equivalent to

$$
Y_{E}\left(\tau_{2} ; \tau_{1}, \tau_{2}\right) 1_{t<\tau_{2}}=D_{t} F^{E}\left(\tau_{2} ; \tau_{1}, \tau_{2}\right) \sigma_{E}^{-1}\left(t ; \tau_{1}, \tau_{2}\right) Y_{E}\left(t ; \tau_{1}, \tau_{2}\right)
$$

Multiply both sides by a square function which integrates to 1 on $\left[0, \tau_{2}\right]$

$$
Y_{E}\left(\tau_{2} ; \tau_{1}, \tau_{2}\right)=\int_{0}^{\tau_{2}} D_{t} F^{E}\left(\tau_{2} ; \tau_{1}, \tau_{2}\right) a(t) \sigma_{E}^{-1}\left(t ; \tau_{1}, \tau_{2}\right) Y_{E}\left(t ; \tau_{1}, \tau_{2}\right) d t .
$$

Now

$$
\begin{aligned}
\Delta_{E}:= & \frac{\partial}{\partial F^{E}\left(0 ; \tau_{1}, \tau_{2}\right)} \mathbb{E}^{Q}\left[g\left(F^{E}\left(\tau_{2} ; \tau_{1}, \tau_{2}\right)\right) h\left(F^{I}\left(\tau_{2} ; \tau_{1}, \tau_{2}\right)\right)\right] \\
= & \mathbb{E}^{Q}\left[g^{\prime}\left(F^{E}\left(\tau_{2} ; \tau_{1}, \tau_{2}\right)\right) h\left(F^{I}\left(\tau_{2} ; \tau_{1}, \tau_{2}\right)\right) \frac{\partial F^{E}\left(\tau_{2} ; \tau_{1}, \tau_{2}\right)}{\partial F^{E}\left(0 ; \tau_{1}, \tau_{2}\right)}\right] \\
= & \mathbb{E}^{\mathbb{Q}}\left[g^{\prime}\left(F^{E}\left(\tau_{2} ; \tau_{1}, \tau_{2}\right)\right) h\left(F^{I}\left(\tau_{2} ; \tau_{1}, \tau_{2}\right)\right) Y_{E}\left(\tau_{2} ; \tau_{1}, \tau_{2}\right)\right] \\
= & \mathbb{E}^{\mathbb{Q}}\left[\int_{0}^{\tau_{2}} g^{\prime}\left(F^{E}\left(\tau_{2} ; \tau_{1}, \tau_{2}\right)\right) h\left(F^{I}\left(\tau_{2} ; \tau_{1}, \tau_{2}\right)\right)\right. \\
& \left.\quad \times D_{t} F^{E}\left(\tau_{2} ; \tau_{1}, \tau_{2}\right) a(t) \sigma_{E}^{-1}\left(t ; \tau_{1}, \tau_{2}\right) Y_{E}\left(t ; \tau_{1}, \tau_{2}\right) d t\right] \\
= & \mathbb{E}^{\mathbb{Q}}\left[h\left(F^{I}\left(\tau_{2} ; \tau_{1}, \tau_{2}\right)\right) \int_{0}^{\tau_{2}} D_{t} g\left(F^{E}\left(\tau_{2} ; \tau_{1}, \tau_{2}\right)\right) a(t) \sigma_{E}^{-1}\left(t ; \tau_{1}, \tau_{2}\right) Y_{E}\left(t ; \tau_{1}, \tau_{2}\right) d t\right] \\
= & \mathbb{E}^{\mathbb{Q}}\left[g\left(F^{E}\left(\tau_{2} ; \tau_{1}, \tau_{2}\right)\right) h\left(F^{I}\left(\tau_{2} ; \tau_{1}, \tau_{2}\right)\right) \int_{0}^{\tau_{2}} a(t)\left(\sigma_{E}^{-1}\left(t ; \tau_{1}, \tau_{2}\right) Y_{E}\left(t ; \tau_{1}, \tau_{2}\right)\right)^{*} d W^{E}(t)\right]
\end{aligned}
$$

where $g^{\prime}$ denotes the derivative of $g$ with respect to $F^{E}\left(0 ; \tau_{1}, \tau_{2}\right)$. Here, we have used the chain rule property, (Property P1), the integration by parts formula (Property P3), and the fact that the Skorohod integral coincides with the Itô stochastic integral (Property P4). Since a continuously differentiable function is dense in $L^{2}$, the result hold for any $g \in L^{2}$ (see Fournié et al. [12] for details).

Similarly, we obtain the following result.

Proposition 4.2. Assume that the diffusion matrix $\sigma_{I}$ is uniformly elliptic. Then for all $a \in \Upsilon_{n}$, the delta of the energy temperature is given by:

$$
\Delta_{I}=\mathbb{E}^{\mathbb{Q}}\left[g\left(F^{E}\left(\tau_{2} ; \tau_{1}, \tau_{2}\right)\right) h\left(F^{I}\left(\tau_{2} ; \tau, \tau_{2}\right)\right) \pi^{\Delta_{I}}\right],
$$

where the Malliavin weight $\pi^{\Delta_{I}}$ is

$$
\pi^{\Delta_{I}}=\int_{0}^{\tau_{2}} a(t)\left(\sigma_{I}^{-1}\left(t ; \tau_{1}, \tau_{2}\right) Y_{I}\left(t ; \tau_{1}, \tau_{2}\right)\right)^{*} d W^{I}(t)
$$

Proof. Follows along the lines of the proof of Proposition of 4.1 .

The following result gives the cross-gamma hedge in the independent case:

Proposition 4.3. Assume that the diffusion matrices $\sigma_{i}, i=E, I$ are uniformly elliptic. Then for all $a \in \Upsilon_{n}$, the following hold:

$$
\Delta_{E I}=\mathbb{E}^{\mathbb{Q}}\left[g\left(F^{E}\left(\tau_{2} ; \tau_{1}, \tau_{2}\right)\right) h\left(F^{I}\left(\tau_{2} ; \tau, \tau_{2}\right)\right) \pi^{\Delta_{E I}}\right]
$$


where the Malliavin weight $\pi^{\Delta_{E I}}$ is

$\pi^{\Delta_{E I}}=\int_{0}^{\tau_{2}} a(t)\left(\sigma_{E}^{-1}\left(t ; \tau_{1}, \tau_{2}\right) Y_{E}\left(t ; \tau_{1}, \tau_{2}\right)\right)^{*} d W^{E}(t) \int_{0}^{\tau_{2}} a(t)\left(\sigma_{I}^{-1}\left(t ; \tau_{1}, \tau_{2}\right) Y_{I}\left(t ; \tau_{1}, \tau_{2}\right)\right)^{*} d W^{I}(t)$.

Proof. We first assume that $g$ and $h$ are continuously differentiable with bounded derivatives. From Proposition 4.1 we have

$$
\Delta_{E}=\mathbb{E}^{\mathbb{Q}}\left[g\left(F^{E}\left(\tau_{2} ; \tau_{1}, \tau_{2}\right)\right) h\left(F^{I}\left(\tau_{2} ; \tau, \tau_{2}\right)\right) Z^{E}\left(\tau_{2}\right)\right] .
$$

As in Proposition 4.1, we introduce

$$
Y_{I}\left(t ; \tau_{1}, \tau_{2}\right)=\exp \left(-\frac{1}{2} \int_{0}^{t} \sigma_{I}^{2}\left(u ; \tau_{1}, \tau_{2}\right) d u+\int_{0}^{t} \sigma_{I}\left(u ; \tau_{1}, \tau_{2}\right) d W^{I}(u)\right) .
$$

This implies that

$$
F^{I}\left(\tau ; \tau_{1}, \tau_{2}\right)=F^{I}\left(0 ; \tau_{1}, \tau_{2}\right) Y_{I}\left(t ; \tau_{1}, \tau_{2}\right)
$$

An application of Property $P 2$ shows that $F^{I}\left(\tau_{2} ; \tau_{1}, \tau_{2}\right)$ belongs to $\mathbb{D}^{1,2}$ and we have:

$$
D_{t} F^{I}\left(\tau_{2} ; \tau_{1}, \tau_{2}\right)=Y_{I}\left(\tau_{2} ; \tau_{1}, \tau_{2}\right) Y_{I}^{-1}\left(t ; \tau_{1}, \tau_{2}\right) \sigma_{I}\left(t ; \tau_{1}, \tau_{2}\right) 1_{t<\tau_{2}} .
$$

This is equivalent to

$$
Y_{I}\left(\tau_{2} ; \tau_{1}, \tau_{2}\right) 1_{t<\tau_{2}}=D_{t} F^{I}\left(\tau_{2} ; \tau_{1}, \tau_{2}\right) \sigma_{I}^{-1}\left(t ; \tau_{1}, \tau_{2}\right) Y_{I}\left(t ; \tau_{1}, \tau_{2}\right) .
$$

Multiply both sides by a square function which integrates to 1 on $\left[0, \tau_{2}\right]$

$$
Y_{I}\left(\tau_{2} ; \tau_{1}, \tau_{2}\right)=\int_{0}^{\tau_{2}} D_{t} F^{I}\left(\tau_{2} ; \tau_{1}, \tau_{2}\right) a(t) \sigma_{I}^{-1}\left(t ; \tau_{1}, \tau_{2}\right) Y_{I}\left(t ; \tau_{1}, \tau_{2}\right) d t .
$$

Now

$$
\begin{aligned}
\Delta_{E I}:= & \frac{\partial}{\partial F^{I}\left(0 ; \tau_{1}, \tau_{2}\right)} \Delta_{E} \\
= & \frac{\partial}{\partial F^{I}\left(0 ; \tau_{1}, \tau_{2}\right)}\left[\mathbb{E}^{\mathbb{Q}}\left[g\left(F^{E}\left(\tau_{2} ; \tau_{1}, \tau_{2}\right)\right) h\left(F^{I}\left(\tau_{2} ; \tau, \tau_{2}\right)\right) Z^{E}\left(\tau_{2}\right)\right]\right] \\
= & \mathbb{E}^{\mathbb{Q}}\left[g\left(F^{E}\left(\tau_{2} ; \tau_{1}, \tau_{2}\right)\right) Z^{E}\left(\tau_{2}\right) h^{\prime}\left(F^{I}\left(\tau_{2} ; \tau, \tau_{2}\right)\right) \frac{\partial F^{I}\left(\tau_{2} ; \tau_{1}, \tau_{2}\right)}{\partial F^{I}\left(0 ; \tau_{1}, \tau_{2}\right)}\right] \\
= & \mathbb{E}^{\mathbb{Q}}\left[g\left(F^{E}\left(\tau_{2} ; \tau_{1}, \tau_{2}\right)\right) Z^{E}\left(\tau_{2}\right) h^{\prime}\left(F^{I}\left(\tau_{2} ; \tau, \tau_{2}\right)\right) Y_{I}\left(\tau_{2} ; \tau_{1}, \tau_{2}\right)\right] \\
= & \mathbb{E}^{\mathbb{Q}}\left[\int_{0}^{\tau_{2}} g\left(F^{E}\left(\tau_{2} ; \tau_{1}, \tau_{2}\right)\right) h^{\prime}\left(F^{I}\left(\tau_{2} ; \tau_{1}, \tau_{2}\right)\right) Z^{E}\left(\tau_{2}\right)\right. \\
& \left.\quad \times D_{t} F^{I}\left(\tau_{2} ; \tau_{1}, \tau_{2}\right) a(t) \sigma_{I}^{-1}\left(t ; \tau_{1}, \tau_{2}\right) Y_{I}\left(t ; \tau_{1}, \tau_{2}\right) d t\right] \\
= & \mathbb{E}^{\mathbb{Q}}\left[g\left(F^{E}\left(\tau_{2} ; \tau_{1}, \tau_{2}\right)\right) Z^{E}\left(\tau_{2}\right)\right. \\
& \left.\quad \times \int_{0}^{\tau_{2}} D_{t}\left(h\left(F^{I}\left(\tau_{2} ; \tau_{1}, \tau_{2}\right)\right)\right) a(t) \sigma_{I}^{-1}\left(t ; \tau_{1}, \tau_{2}\right) Y_{I}\left(t ; \tau_{1}, \tau_{2}\right) d t\right] \\
= & \mathbb{E}^{\mathbb{Q}}\left[g\left(F^{E}\left(\tau_{2} ; \tau_{1}, \tau_{2}\right)\right) h\left(F^{I}\left(\tau_{2} ; \tau_{1}, \tau_{2}\right)\right) Z^{E}\left(\tau_{2}\right)\right. \\
& \left.\quad \times \int_{0}^{\tau_{2}} a(t)\left(\sigma_{I}^{-1}\left(t ; \tau_{1}, \tau_{2}\right) Y_{I}\left(t ; \tau_{1}, \tau_{2}\right)\right)^{*} d W^{I}(t)\right] .
\end{aligned}
$$


Here, we have used the chain rule property, (Property P1), the integration by parts formula (Property P3), and the fact that the Skorohod integral coincides with the Itô stochastic integral (Property P4).

The result can be extended to the general case by a density argument. We omit the details.

\section{The Correlation Case}

We consider the following HJM

$$
\begin{aligned}
& d F^{E}\left(t ; \tau_{1}, \tau_{2}\right)=\sigma_{E}\left(t, F^{E}\left(t ; \tau_{1}, \tau_{2}\right)\right) d W^{E}(t) \\
& d F^{I}\left(t ; \tau_{1}, \tau_{2}\right)=\rho \sigma_{I}\left(t, F^{I}\left(t ; \tau_{1}, \tau_{2}\right)\right) d W^{E}(t)+\sigma_{I}\left(t, F^{I}\left(t ; \tau_{1}, \tau_{2}\right)\right) \sqrt{1-\rho^{2}} d \widetilde{W}^{I}(t) .
\end{aligned}
$$

with $F^{E}\left(0 ; \tau_{1}, \tau_{2}\right)>0$, and $F^{I}(0 ; \cdot)>0$.

That is, we consider the case when there is correlation between $F^{E}$ and $F^{I}$. Suppose the Brownian motions $B_{1}$ and $B_{2}$ are independent. Let $W_{1}=B_{1}$ and $W_{2}=\rho B_{1}+\sqrt{1-\rho^{2}} B_{2}$. This implies that

$$
g\left(W_{1}\right) h\left(W_{2}\right)=g\left(B_{1}\right) h\left(\rho B_{1}+\sqrt{1-\rho^{2}} B_{2}\right) .
$$

In this setting, we have the following quanto option structure:

$$
C=\mathbb{E}^{\mathbb{Q}}\left[g\left(F^{E}\left(\tau_{2} ; \tau_{1}, \tau_{2}\right)\right) h\left(\rho F^{E}\left(\tau_{2} ; \tau_{1}, \tau_{2}\right)+\sqrt{1-\rho^{2}} F^{I}\left(\tau_{2} ; \tau_{1}, \tau_{2}\right)\right)\right] .
$$

Now we derive the energy delta.

Proposition 5.1. Assume that the diffusion matrix $\sigma_{E}$ is uniformly elliptic. Then for all $a \in \Upsilon_{n}$, the following hold:

$$
\Delta_{E}=\mathbb{E}^{\mathbb{Q}}\left[g\left(F^{E}\left(\tau_{2} ; \tau_{1}, \tau_{2}\right)\right) h\left(\rho F^{E}\left(\tau_{2} ; \tau_{1}, \tau_{2}\right)+\sqrt{1-\rho^{2}} F^{I}\left(\tau_{2} ; \tau_{1}, \tau_{2}\right)\right) \pi^{\Delta_{E}}(1+\rho)\right],
$$

where the Malliavin weight $\pi^{\Delta_{E}}$ is

$$
\pi^{\Delta_{E}}=\int_{0}^{\tau_{2}} a(t)\left(\sigma_{E}^{-1}\left(t ; \tau_{1}, \tau_{2}\right) Y_{E}\left(t ; \tau_{1}, \tau_{2}\right)\right)^{*} d W^{E}(t)
$$

Proof. Let $g$ be a continuously differentiable function with bounded derivatives. As in Proposition 4.1, introduce

$$
Y_{E}\left(t ; \tau_{1}, \tau_{2}\right)=\exp \left(-\frac{1}{2} \int_{0}^{t} \sigma_{E}^{2}\left(u ; \tau_{1}, \tau_{2}\right) d u+\int_{0}^{t} \sigma_{E}\left(u ; \tau_{1}, \tau_{2}\right) d W^{E}(u)\right) .
$$

This implies that

$$
F^{E}\left(t ; \tau_{1}, \tau_{2}\right)=F^{E}\left(0 ; \tau_{1}, \tau_{2}\right) Y_{E}\left(t ; \tau_{1}, \tau_{2}\right)
$$

An application of Property $P 2$ shows that $F^{E}\left(\tau_{2} ; \tau_{1}, \tau_{2}\right)$ belongs to $\mathbb{D}^{1,2}$ and we have:

$$
D_{t} F^{E}\left(\tau_{2} ; \tau_{1}, \tau_{2}\right)=Y_{E}\left(\tau_{2} ; \tau_{1}, \tau_{2}\right) Y_{E}^{-1}\left(t ; \tau_{1}, \tau_{2}\right) \sigma_{E}\left(t ; \tau_{1}, \tau_{2}\right) 1_{t<\tau_{2}} .
$$

This is equivalent to

$$
Y_{E}\left(\tau_{2} ; \tau_{1}, \tau_{2}\right) 1_{t<\tau_{2}}=D_{t} F^{E}\left(\tau_{2} ; \tau_{1}, \tau_{2}\right) \sigma_{E}^{-1}\left(t ; \tau_{1}, \tau_{2}\right) Y_{E}\left(t ; \tau_{1}, \tau_{2}\right)
$$


Multiply both sides by a square function which integrates to 1 on $\left[0, \tau_{2}\right]$

$$
Y_{E}\left(\tau_{2} ; \tau_{1}, \tau_{2}\right)=\int_{0}^{\tau_{2}} D_{t} F^{E}\left(\tau_{2} ; \tau_{1}, \tau_{2}\right) a(t) \sigma_{E}^{-1}\left(t ; \tau_{1}, \tau_{2}\right) Y_{E}\left(t ; \tau_{1}, \tau_{2}\right) d t
$$

Now

$$
\begin{aligned}
\Delta_{E}=\mathbb{E}^{\mathbb{Q}} & {\left[g^{\prime}\left(F^{E}\left(\tau_{2} ; \tau_{1}, \tau_{2}\right)\right) h\left(\rho F^{E}\left(\tau_{2} ; \tau_{1}, \tau_{2}\right)+\sqrt{1-\rho^{2}} F^{I}\left(\tau_{2} ; \tau_{1}, \tau_{2}\right)\right) Y_{E}\left(\tau_{2} ; \tau_{1}, \tau_{2}\right)\right.} \\
& \left.+g\left(F^{E}\left(\tau_{2} ; \tau_{1}, \tau_{2}\right)\right) h^{\prime}\left(\rho F^{E}\left(\tau_{2} ; \tau_{1}, \tau_{2}\right)+\sqrt{1-\rho^{2}} F^{I}\left(\tau_{2} ; \tau_{1}, \tau_{2}\right)\right) \rho Y_{E}\left(\tau_{2} ; \tau_{1}, \tau_{2}\right)\right] \\
=\mathbb{E}^{\mathbb{Q}} & {\left[\int_{0}^{\tau_{2}} g^{\prime}\left(F^{E}\left(\tau_{2} ; \tau_{1}, \tau_{2}\right)\right) h\left(\rho F^{E}\left(\tau_{2} ; \tau_{1}, \tau_{2}\right)+\sqrt{1-\rho^{2}} F^{I}\left(\tau_{2} ; \tau_{1}, \tau_{2}\right)\right)\right.} \\
& \times D_{t} F^{E}\left(\tau_{2} ; \tau_{1}, \tau_{2}\right) a(t) \sigma_{E}^{-1}\left(t ; \tau_{1}, \tau_{2}\right) Y_{E}\left(t ; \tau_{1}, \tau_{2}\right) d t \\
& +\int_{0}^{\tau_{2}} g\left(F^{E}\left(\tau_{2} ; \tau_{1}, \tau_{2}\right)\right) h^{\prime}\left(\rho F^{E}\left(\tau_{2} ; \tau_{1}, \tau_{2}\right)+\sqrt{1-\rho^{2}} F^{I}\left(\tau_{2} ; \tau_{1}, \tau_{2}\right)\right) \\
& \left.\times D_{t} F^{E}\left(\tau_{2} ; \tau_{1}, \tau_{2}\right) a(t) \sigma_{E}^{-1}\left(t ; \tau_{1}, \tau_{2}\right) Y_{E}\left(t ; \tau_{1}, \tau_{2}\right) d t\right] \\
=\mathbb{E}^{\mathbb{Q}}[ & h\left(\rho F^{E}\left(\tau_{2} ; \tau_{1}, \tau_{2}\right)+\sqrt{1-\rho^{2}} F^{I}\left(\tau_{2} ; \tau_{1}, \tau_{2}\right)\right) \\
\times & \int_{0}^{\tau_{2}} D_{t} g\left(F^{E}\left(\tau_{2} ; \tau_{1}, \tau_{2}\right)\right) a(t) \sigma_{E}^{-1}\left(t ; \tau_{1}, \tau_{2}\right) Y_{E}\left(t ; \tau_{1}, \tau_{2}\right) d t+\rho g\left(F^{E}\left(\tau_{2} ; \tau_{1}, \tau_{2}\right)\right) \\
& \left.\times \int_{0}^{\tau_{2}} D_{t} h\left(\rho F^{E}\left(\tau_{2} ; \tau_{1}, \tau_{2}\right)+\sqrt{1-\rho^{2}} F^{I}\left(\tau_{2} ; \tau_{1}, \tau_{2}\right)\right) a(t) \sigma_{E}^{-1}\left(t ; \tau_{1}, \tau_{2}\right) Y_{E}\left(t ; \tau_{1}, \tau_{2}\right) d t\right] \\
=\mathbb{E}^{\mathbb{Q}} & {\left[h\left(\rho F^{E}\left(\tau_{2} ; \tau_{1}, \tau_{2}\right)+\sqrt{1-\rho^{2}} F^{I}\left(\tau_{2} ; \tau_{1}, \tau_{2}\right)\right)\right.} \\
& \times g\left(F^{E}\left(\tau_{2} ; \tau_{1}, \tau_{2}\right)\right) \int_{0}^{\tau_{2}} a(t)\left(\sigma_{E}^{-1}\left(t ; \tau_{1}, \tau_{2}\right) Y_{E}\left(t ; \tau_{1}, \tau_{2}\right)\right)^{*} d W^{E}(t) \\
& +\rho g\left(F^{E}\left(\tau_{2} ; \tau_{1}, \tau_{2}\right)\right) h\left(\rho F^{E}\left(\tau_{2} ; \tau_{1}, \tau_{2}\right)+\sqrt{1-\rho^{2}} F^{I}\left(\tau_{2} ; \tau_{1}, \tau_{2}\right)\right) \\
& \left.\times \int_{0}^{\tau_{2}} a(t)\left(\sigma_{E}^{-1}\left(t ; \tau_{1}, \tau_{2}\right) Y_{E}\left(t ; \tau_{1}, \tau_{2}\right)\right)^{*} d W^{E}(t)\right] . \\
& \quad
\end{aligned}
$$

As in the proof of Proposition 4.1, we have used the chain rule property, (Property P1), the integration by parts formula (Property P3), and the fact that the Skorohod integral coincides with the Itô stochastic integral (Property P4).

The result can be extended to the general case by a density argument. We omit the details.

Now we derive the temperature delta in the correlation case.

Proposition 5.2. Assume that the diffusion matrix $\sigma_{I}$ is uniformly elliptic. Then for all $a \in \Upsilon_{n}$, the following hold :

$$
\Delta_{I}=\sqrt{1-\rho^{2}} \mathbb{E}^{\mathbb{Q}}\left[g\left(F^{E}\left(\tau_{2} ; \tau_{1}, \tau_{2}\right)\right) h\left(\rho F^{E}\left(\tau_{2} ; \tau_{1}, \tau_{2}\right)+\sqrt{1-\rho^{2}} F^{I}\left(\tau_{2} ; \tau_{1}, \tau_{2}\right)\right) \pi^{\Delta_{I}}\right] .
$$

where the Malliavin weight $\pi^{\Delta_{I}}$ is

$$
\pi^{\Delta_{I}}=\int_{0}^{\tau_{2}} a(t)\left(\sigma_{I}^{-1}\left(t ; \tau_{1}, \tau_{2}\right) Y_{I}\left(t ; \tau_{1}, \tau_{2}\right)\right)^{*} d W^{I}(t)
$$


Proof. The proof is similar to that of Proposition 5.1.

The following result gives the cross-gamma hedge in the correlation case.

Proposition 5.3. Assume that the diffusion matrices $\sigma_{i}, i=E, I$, are uniformly elliptic. Then for all $a \in \Upsilon_{n}$, the following hold:

$$
\begin{aligned}
\Delta_{E I}= & \sqrt{1-\rho^{2}} \mathbb{E}^{\mathbb{Q}}\left[g\left(F^{E}\left(\tau_{2} ; \tau_{1}, \tau_{2}\right)\right) h\left(\rho F^{E}\left(\tau_{2} ; \tau_{1}, \tau_{2}\right)+\sqrt{1-\rho^{2}} F^{I}\left(\tau_{2} ; \tau_{1}, \tau_{2}\right)\right) \pi^{\Delta_{E I}}\right. \\
& \left.+\rho \sqrt{1-\rho^{2}} g\left(F^{E}\left(\tau_{2} ; \tau_{1}, \tau_{2}\right)\right) h\left(\rho F^{E}\left(\tau_{2} ; \tau_{1}, \tau_{2}\right)+\sqrt{1-\rho^{2}} F^{I}\left(\tau_{2} ; \tau_{1}, \tau_{2}\right)\right) \pi^{\Delta_{E I}}\right]
\end{aligned}
$$

where the Malliavin weight $\pi^{\Delta_{E I}}$ is:

$\pi^{\Delta_{E I}}=\int_{0}^{\tau_{2}} a(t)\left(\sigma_{E}^{-1}\left(t ; \tau_{1}, \tau_{2}\right) Y_{E}\left(t ; \tau_{1}, \tau_{2}\right)\right)^{*} d W^{E}(t) \int_{0}^{\tau_{2}} a(t)\left(\sigma_{I}^{-1}\left(t ; \tau_{1}, \tau_{2}\right) Y_{I}\left(t ; \tau_{1}, \tau_{2}\right)\right)^{*} d W^{I}(t)$.

Proof. The proof follows the same line of argument as in the proof of Proposition 4.3. The details are omitted.

5.1. The Residual Risk. If we take the independent delta of energy $\Delta^{\text {Ind }}$, say, as the benchmark value and the correlated case as $\Delta^{\text {Corr }}$. Then the residual risk is determined by the difference between the independent delta of energy and the correlated case as follows:

$$
\left|\Delta^{\text {Corr }}-\Delta^{\text {Ind }}\right|
$$

for each $\rho$. The same analysis goes for the cross-gamma formulae.

\section{EXAMPLES}

We will provide Malliavin weights in the case where the quanto option payoff functions depend on the terminal value, that is, $\tau_{2}=T$

6.1. The independent case. We consider the following stochastic differential equations to describe the energy price $F^{E}$ and the temperature price $F^{I}$ dynamics

$$
\begin{aligned}
\frac{d F^{E}}{F^{E}} & =\sigma_{E} d W_{t}^{E}, F^{E}(0)>0 \\
\frac{d F^{I}}{F^{I}} & =\sigma_{I} d W_{t}^{I}, F^{I}(0)>0,
\end{aligned}
$$

where $\sigma_{E}, \sigma_{I}$ are deterministic volatilities and $W^{E}, W^{I}$ are independent Brownian motions. The quanto option pricing formula is then expressed as

$$
C_{t}=\mathbb{E}^{\mathbb{Q}}\left[g\left(F^{E}\right) h\left(F^{I}\right)\right] .
$$

By using the general formulae developed in the previous sections, we are able to compute analytically the values of different Malliavin weights. Here we set $a(t)=\frac{1}{T}$. We have

$$
\pi^{\Delta_{E}}=\frac{1}{F^{E}(0) T} \int_{0}^{T} \frac{1}{\sigma_{E}} d W^{E}(t) .
$$




$$
\begin{gathered}
\pi^{\Delta_{I}}=\frac{1}{F^{I}(0) T} \int_{0}^{T} \frac{1}{\sigma_{I}} d W^{I}(t) . \\
\pi^{\Delta_{E I}}=\frac{1}{F^{E}(0) F^{I}(0) T^{2}}\left(\int_{0}^{T} \frac{1}{\sigma_{E}} d W^{E}(t)\right)\left(\int_{0}^{T} \frac{1}{\sigma_{I}} d W^{I}(t)\right) .
\end{gathered}
$$

6.2. The correlation case. Again, we consider the following stochastic differential equations to describe the energy price $F^{E}$ and the temperature price $F^{I}$ dynamics

$$
\begin{aligned}
& \frac{d F^{E}}{F^{E}}=\sigma_{E} d W_{t}^{E}, F^{E}(0)>0 \\
& \frac{d F^{I}}{F^{I}}=\rho \sigma_{I} d W_{t}^{I}+\sigma_{I} \sqrt{1-\rho^{2}} d \widetilde{W}^{I}, F^{I}(0)>0,
\end{aligned}
$$

where $W_{t}^{E}, W_{t}^{I}$ are correlated Brownian motions with correlation parameter $\rho \in(-1,1)$. The system of stochastic differential equations can be written in a matrix form

$$
\left(\begin{array}{c}
d F^{E} \\
d F^{I}
\end{array}\right)=\left(\begin{array}{cc}
\sigma_{E} F^{E} & 0 \\
\rho \sigma_{I} F^{I} & \sigma_{I} \sqrt{1-\rho^{2}} F^{I}
\end{array}\right)\left(\begin{array}{c}
d W^{E} \\
d \widetilde{W}^{I}
\end{array}\right) .
$$

The inverse matrix of

$$
\left(\begin{array}{cc}
\sigma_{E} F^{E} & 0 \\
\rho \sigma_{I} F^{I} & \sigma_{I} \sqrt{1-\rho^{2}} F^{I}
\end{array}\right)
$$

is calculated as

$$
\frac{1}{\sigma_{E} \sigma_{I} \sqrt{1-\rho^{2}} F^{E} F^{I}}\left(\begin{array}{cc}
\sigma_{I} \sqrt{1-\rho^{2}} F^{I} & 0 \\
-\rho \sigma_{I} F^{I} & \sigma_{E} F^{E}
\end{array}\right)=\left(\begin{array}{cc}
\frac{1}{\sigma_{E} F^{E}} & 0 \\
-\frac{\rho}{\sigma_{E} \sqrt{1-\rho^{2}} F^{E}} & \frac{1}{\sigma_{I} \sqrt{1-\rho^{2}} F^{I}}
\end{array}\right) .
$$

The quanto option pricing formula, in this setting, is given by

$$
C_{t}=\mathbb{E}^{\mathbb{Q}}\left[g\left(F^{E}\right) h\left(\rho F^{E}+\sqrt{1-\rho^{2}} F^{I}\right)\right] .
$$

By using the general formulae developed in the previous sections, we are able to compute analytically the values of different Malliavin weights. Here we set $a(t)=\frac{1}{T}$. We have

$$
\begin{gathered}
\pi^{\Delta_{E}}=\frac{1}{F^{E}(0) T} \int_{0}^{T} \frac{1}{\sigma_{E}} d W^{E}(t)-\frac{1}{F^{E}(0) T} \int_{0}^{T} \frac{\rho}{\sigma_{E} \sqrt{1-\rho^{2}}} d \widetilde{W}^{I}(t) . \\
\pi^{\Delta_{I}}=\frac{1}{F^{I}(0) T} \int_{0}^{T} \frac{1}{\sigma_{I} \sqrt{1-\rho^{2}}} d \widetilde{W}^{I}(t) . \\
\pi^{\Delta_{E I}}=\frac{1}{F^{E}(0) F^{I}(0) T^{2}}\left(\int_{0}^{T} \frac{1}{\sigma_{E}} d W^{E}(t)-\int_{0}^{T} \frac{\rho}{\sigma_{E} \sqrt{1-\rho^{2}}} d \widetilde{W}^{I}(t)\right)\left(\int_{0}^{T} \frac{1}{\sigma_{I} \sqrt{1-\rho^{2}}} d \widetilde{W}^{I}(t)\right) \\
-\frac{1}{F^{E}(0) F^{I}(0) T^{2}} \int_{0}^{T} \frac{\rho}{\sigma_{E} \sigma_{I}\left(1-\rho^{2}\right)} d t .
\end{gathered}
$$




\section{Concluding Remarks}

In this paper, we have derived the delta, the cross-gamma expectation formulae of the quanto energy option written on a forward contract under the HJM framework. We have considered the independent and the correlation cases to facilitate the residual risk analysis. The results are an extension of the work in Benth et al. [1] as they accommodate discontinuous payoff functions. It will be interesting to consider the case with stochastic volatility with a positive Lévy processes when the market is incomplete. In Benth et al. [3], the authors analysed such a volatility model for a different payoff structure to the one considered in this paper. This will be considered in future research.

Acknowledgment. The authors would like to thank Prof Fred E. Benth for discussing the modelling issues in this paper. The work of R. K. was supported in part by the National Research Foundation of South Africa (Project No. 90313). The work of F. J. M. was supported in part by the National Research Foundation of South Africa (Grant Number: 105924).

\section{REFERENCES}

[1] Benth, F. E.; Lange, N.; Myklebust, T. A. Pricing and hedging quanto options in energy markets. The Journal of Energy Markets. 2015, 8.1, 1-35.

[2] Nualart, D. The Malliavin Calculus and Related Topics, Springer, 2006.

[3] Benth, F. E.; Groth, M.; Wallin, O. Derivative-free Greeks for the Barndorff-Nielsen and Shephard stochastic volatility model. Stochastics: An International Journal of Probability and Stochastic Processes. 2010, 82, 3, 291-313.

[4] Caporin, M.;J. Prés, J.; Toppo, H. Model based Monte-Carlo pricing of energy and temperature quanto options and hedging quanto options in energy markets. The Journal of Energy Economics. 2012, 34.5 : 1700-1712.

[5] Ho, T. S.; Stapleton, R. C.; Subrabmanyan, M. G. Correlation risk, cross-market derivative products and portfolio performance. Journal of European Financial Management. 1995, 1 2, 105-122.

[6] Zhang, P. G. Exotic options. A guide to second generation options. World Scientific, Singapore, 2001.

[7] Heath, D.; Jarrow, R.; Merton, A. Bond pricing and term structure of interest rates: a new methodology for contingent claim valuation. Econometrica. 1992, 60, 77-105.

[8] Benth, F. E.; Benth, J. S.; Koekebakker, S. Stochastic modelling of electricity and related markets. World Scientific, 2008.

[9] Clewlow, L.; Strickland, C. Energy derivatives: Pricing and risk management, Lacima Publications, 2000.

[10] Benth, F. E; Dahl, L. O.; Kalsen, K. H. Quasi Monte-Carlo evaluation of sensitivities of options in commodity and energy markets. Int. J. Theor. Appl. Finance. 2003, Vol 6 8, pp. 865-884.

[11] Di Nunno, G.; Oksendal, B.; Proske, F. Malliavin calculus for Lévy processes with applications to finance. Springer - New York, 2009.

[12] Fournié, E; Lasry, J. M.; Lebuchoux, J; Lions, P. L.; Touzi, N. Applications of Malliavin calculus to Monte-Carlo methods in finance. 1999, Finance Stochastic 3, 391-412.

[13] Fournié, E; Lasry, J. M.; Lebuchoux, J; Lions, P. L. Applications of Malliavin calculus to Monte-Carlo methods in finance. Finance Stochastic. 2001, 5, 201-236.

[14] Karatzas, I.; D. Ocone D. A generalized Clark representation formula, with application to optimal portfolios. Stochastics and stochastic reports, 1991, 34, pp. 187-220.

[15] Mhlanga, F. J. Computation of Greeks using Malliavin calculus. PhD Thesis. University of Cape Town, South Africa, 2011. 
Department of Mathematics and Applied Mathematics, University of Pretoria, 0002, South AFrica

E-mail address: rodwell.kufakunesu@up.ac.za

Department of Mathematics and Applied Mathematics, University of Limpopo, Private Bag X1106, Sovenga, 0727, South Africa

E-mail address: farai.mlanga@ul.ac.za 